\title{
Coexisting high-grade glandular and squamous cervical lesions and human papillomavirus infections
}

\author{
RLM Bekkers*,', J Bulten ${ }^{2}$, A Wiersma-van Tilburg ${ }^{3}$, M Mravunac ${ }^{4}$, CPT Schijf', LFAG Massuger', WGV Quint ${ }^{5}$ \\ and WJG Melchers ${ }^{6}$
}

'Department of Gynecology/Obstetrics, University Medical Center Nijmegen, St Radboud, PO Box 9101, 6500 HB Nijmegen, The Netherlands; ${ }^{2}$ Department of Pathology, University Medical Center Nijmegen, St Radboud, PO Box 9101, 6500 HB Nijmegen, The Netherlands; ${ }^{3}$ Department of Pathology, Rijnstate Hospital Arnhem, Arnhem, The Netherlands; ${ }^{4}$ Department of Pathology, Canisius Wilhelmina Hospital Nijmegen, Nijmegen, The Netherlands; ${ }^{5}$ Delft Diagnostic Laboratory, Delft, The Netherlands; ${ }^{6}$ Department of Medical Microbiology, University Medical Center Nijmegen, St Radboud, PO Box 9101, 6500 HB Nijmegen, The Netherlands

The frequency of high-risk human papillomavirus (hr-HPV) genotypes in patients with adenocarcinoma in situ (ACIS) with coexisting cervical intraepithelial neoplasia (CIN), ACIS without coexisting CIN, and high-grade CIN (CIN II/III) was studied, in order to gain more insight into the relation between hr-HPV infections and the development of coexisting squamous and glandular lesions. The $\mathrm{SPF}_{10}$ LiPA PCR was used to detect simultaneously 25 different HPV genotypes in biopsies obtained from 90 patients with CIN II/III, 47 patients with ACIS without coexisting CIN, and 49 patients with ACIS and coexisting CIN. hr-HPV was detected in 84 patients (93\%) with CIN II/III, 38 patients (8I\%) with ACIS without CIN, and in 47 patients (96\%) with ACIS and coexisting CIN. A total of I3 different hr-HPV genotypes were detected in patients with CIN II/III, and only five in patients with ACIS with/without coexisting CIN HPV 31, multiple hr-HPV genotypes, and HPV genotypes other than 16, I 8, and 45 were significantly more often detected in patients with CIN II/III, while HPV I 8 was significantly more often detected in patients with ACIS with/without CIN. There were no significant differences in the frequency of specific hr-HPV genotypes between patients with ACIS with or without coexisting CIN. In conclusion, the frequency of specific hr-HPV genotypes is similar for patients with ACIS without CIN and patients with ACIS and coexisting CIN, but is significantly different for patients with CIN II/III without ACIS. These findings suggest that squamous lesions, coexisting with highgrade glandular lesions, are aetiologically different from squamous lesions without coexisting glandular lesions.

British Journal of Cancer (2003) 89, 886-890. doi:I0.I038/sj.bjc.660I 204 www.bjcancer.com

(c) 2003 Cancer Research UK

Keywords: ACIS; Coexisting CIN; HPV genotypes

A causal relation between high-risk human papillomavirus (hr-HPV) infections and cervical cancer has been documented in the literature beyond reasonable doubt (Bosch et al, 2002; Wright et al, 2002). High-risk human papillomavirus can be detected in almost $100 \%$ of squamous carcinomas and adenocarcinomas of the uterine cervix (Walboomers et al, 1999; Pirog et al, 2000). Numerous studies have suggested that the development of squamous cervical carcinoma is preceded by cervical intraepithelial neoplasia (CIN) (Wright et al, 2002; Bosch et al, 2002).

The frequency of hr-HPV genotypes in CIN lesions has been studied previously and its detection rate rises with increasing severity of the CIN lesion (Bekkers et al, 2002a; Bosch et al, 2002; Wright et al, 2002). Only limited studies are available on the frequency of hr-HPV genotypes in premalignant glandular lesions and the number of patients in most studies is rather low (see Table 1) (Colgan and Lickrish, 1990; Leary et al, 1991; Higgins et al, 1992; Duggan et al, 1995; Anciaux et al, 1997; McLachlin et al, 1997; Pirog et al, 2000; Riethdorf et al, 2000, 2002; Madeleine et al, 2001). High-risk human papillomavirus

*Correspondence: Dr RLM Bekkers; E-mail: R.Bekkers@obgyn.umcn.nl Received 14 March 2003; revised 16 June 2003; accepted 24 June 2003 was detected in $66-100 \%$ of patients with ACIS (Anciaux et al, 1997; Riethdorf et al, 2000). Approximately $48 \%$ of all women diagnosed with ACIS have coexisting squamous lesions (Colgan and Lickrish, 1990; Leary et al, 1991; Higgins et al, 1992; Duggan et al, 1995; Anciaux et al, 1997; McLachlin et al, 1997; Pirog et al, 2000; Riethdorf et al, 2000, 2002; Madeleine et al, 2001), but the frequency of specific hr-HPV genotypes in patients with ACIS has not been studied in relation with the presence or absence of coexisting CIN lesions. In this study, the frequency of specific hr-HPV genotypes in patients with ACIS and coexisting CIN is compared with its frequency in patients with ACIS without coexisting CIN, and patients with CIN without ACIS, in order to gain more insight into the relation between hr-HPV infections and the development of coexisting squamous and glandular lesions.

\section{MATERIAL AND METHODS}

\section{Glandular lesions}

In the automated databases of the pathology laboratories of the University Medical Center Nijmegen, the Canisius Wilhelmina Hospital Nijmegen, and the Rijnstate Hospital Arnhem, 120 
Table I Summary of all studies since 1990 with more than $20 \mathrm{ACIS}$ patients in relation with hr-HPV and/or coexisting CIN

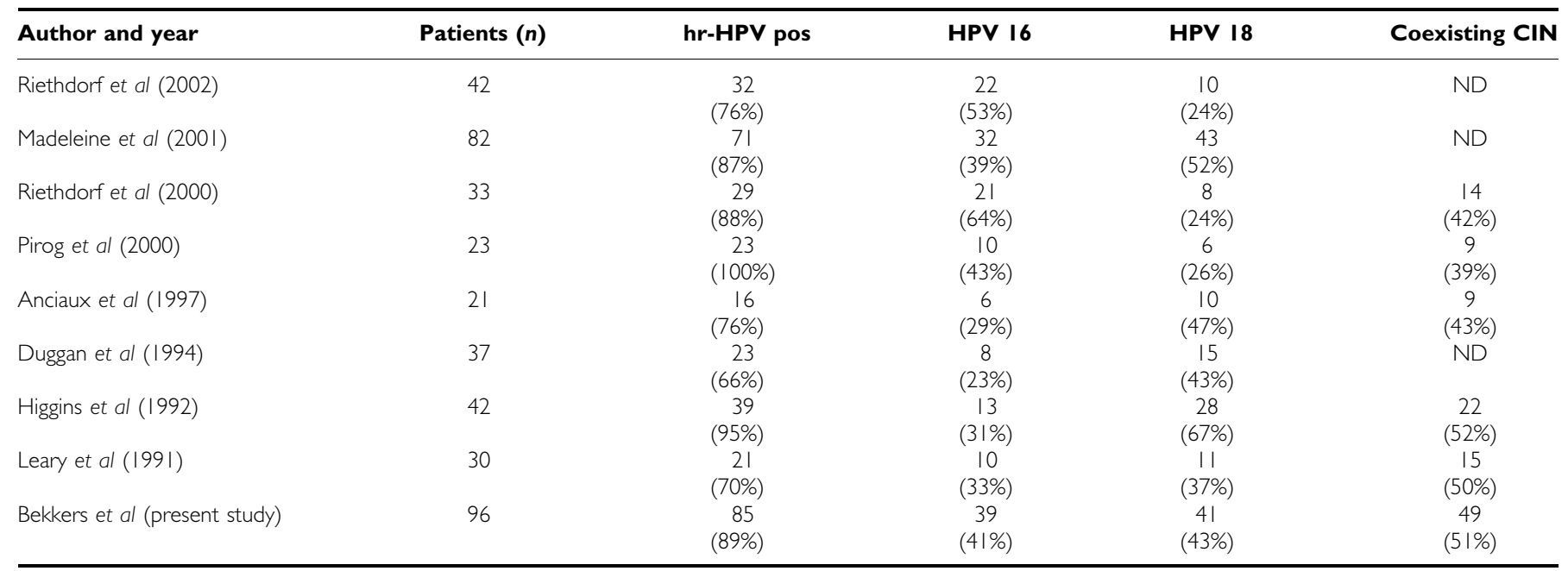

$\mathrm{ACIS}=$ adenocarcinoma in situ; hr-HPV = high-risk human papillomavirus; $\mathrm{CIN}=$ cervical intraepithelial neoplasia; HPV = human papillomavirus; ND = not documented.

patients diagnosed with ACIS between 1988 and 2000 were identified. No histopathological material was left for further study of nine patients, and six patients had a histopathological diagnosis of invasive carcinoma within 1 month of the diagnosis of ACIS. Of the remaining 105 patients, the histopathological slides of a biopsy-, cone-, or hysterectomy specimen were re-examined independently, by three experienced gynaecopathologists (JB, $\mathrm{AWvT}$, and MM). Complete agreement on the diagnosis of ACIS was reached in 96 patients $(91 \%)$, according to the criteria of Brown and Wells. The criteria of Brown and Wells take architectural (glandular irregularity and complexity) and cytological criteria (nuclear enlargement, hyperchromasia, pseudostratification of nuclei, increased or abnormal mitoses) into account (Wells and Brown, 1986). The three pathologists reached no consensus on the diagnosis of ACIS in nine patients, and these patients were excluded.

Coexisting squamous intraepithelial lesions were detected and confirmed by consensus of the three pathologists (JB, AWvT, $\mathrm{MM}$ ) in the slides of 49 of the 96 patients (51\%); 35 patients (71\%) had coexisting CIN 3 (severe dysplasia, or carcinoma in situ), eight patients $(16 \%)$ had coexisting CIN 2 (moderate dysplasia), and six patients (12\%) had coexisting CIN 1 (mild dysplasia). In 41 of these 49 patients (84\%), CIN and ACIS were diagnosed in the same slide/section, and hr-HPV detection was carried out on adjacent sections, containing both the squamous and glandular lesion. In eight patients, CIN was diagnosed in a different slide than in which ACIS was diagnosed and on which hr-HPV detection was performed. Five of these eight patients had coexisting CIN I, two had coexisting CIN II, and one had coexisting CIN III. The mean age of the 96 patients was 38.3 years $(28-73)$ at the time of the diagnosis.

A cervical scrape preceding the diagnosis of ACIS less than 6 months was made in 43 of the 49 patients $(88 \%)$ with ACIS and coexisting CIN, and in 40 of the 47 patients (85\%) with ACIS without coexisting CIN. The cervical scrape indicated a highgrade glandular (moderate atypia-ACIS), a high-grade squamous lesion (moderate dysplasia-CIS), or both in, respectively, 13 (30\%), $15(35 \%)$, and $13(30 \%)$ patients with ACIS and coexisting CIN, and in, respectively, $24(60 \%)$, four (10\%), and eight $(20 \%)$ patients with ACIS without coexisting CIN. The remaining six patients had only mild atypia of glandular cells in the cervical scrape.

\section{Squamous lesions}

A control group of 90 patients consisted of patients who consulted the colposcopy clinic at the UMC between 1997 and 1999 and who were diagnosed with high-grade CIN in the biopsy specimen of a large-loop excision of the transformation zone. All slides were reviewed by an experienced gynaecopathologist (JB), and the diagnosis of high-grade CIN was confirmed. Of these 90 patients, 18 patients $(20 \%)$ were diagnosed with moderate dysplasia (CIN II), 36 patients $(40 \%)$ with severe dysplasia (CIN III), and 36 patients (40\%) with carcinoma in situ (CIN III). The mean age at the time of diagnosis was 37.6 years $(28-59)$.

A cervical scrape preceding the diagnosis of CIN II/III less than 6 months was taken of all 90 patients. The cervical scrape indicated a high-grade squamous lesion in 80 patients $(89 \%)$, a high-grade glandular and squamous lesion in one patient (1\%), and low-grade squamous lesions in nine patients $(10 \%)$.

Taking the preceding cervical scrapes of all patients with ACIS and CIN II/III together, the cervical scrape indicated a high-grade glandular lesion in 37 patients (21\%), a high-grade squamous lesion in 99 patients (57\%), both in 22 patients (13\%), and only low-grade lesions in 15 patients $(9 \%)$.

\section{HPV detection}

A 3- $\mu \mathrm{m}$ section of the histopathological specimens, on which ACIS and/or CIN II/III was diagnosed, was taken for HPV analysis. In 41 of the 49 patients with ACIS and coexisting CIN, HPV detection was carried out on a section containing both the glandular and squamous lesion. HPV detection was performed using a broadspectrum short-fragment polymerase chain reaction $\left(\mathrm{SPF}_{10} \mathrm{PCR}\right)$ as previously described (Kleter et al, 1998, 1999; Melchers et al, 1999; Quint et al, 2001; Bekkers et al, 2002b). In case of a positive PCR, subsequent genotyping was performed via a reverse hybridisation line probe assay (LiPA), allowing for simultaneous typing of $25 \mathrm{HPV}$ genotypes, including hr-HPV genotypes 16, 18, $31,33,35,39,45,51,52,56,58,59,66$, and 68. This $\mathrm{SPF}_{10} \mathrm{LiPA}$ PCR HPV detection method is highly sensitive, specific and reproducible and has been clinically validated (Kleter et al, 1998, 1999; Melchers et al, 1999; Pirog et al, 2000; Quint et al, 2001). If more than one hr-HPV genotype was detected with the $\mathrm{SPF}_{10}$ LiPA PCR in a single sample of an individual patient, that patient was considered to be infected with multiple hr-HPV genotypes. 


\section{Analysis}

The frequency of specific hr-HPV genotypes in patients with ACIS without coexisting CIN, ACIS with coexisting CIN, and CIN II/III without ACIS were compared. The frequency of specific hr-HPV genotypes was also investigated in relation with the suspicion of a high-grade glandular and/or high-grade squamous lesion in the preceding cervical scrape. Statistical analysis including $\chi^{2}$ tests and independent $t$-tests was performed, considering all values of $P<0.05$ to be significant.

\section{RESULTS}

High-risk human papillomavirus genotypes were detected in $93 \%$ of the patients with CIN II/III, $81 \%$ of the patients with ACIS without coexisting CIN, and in $96 \%$ of the patients with ACIS and coexisting CIN. Figure 1 shows that HPV 31 and multiple hr-HPV genotypes were significantly more often detected

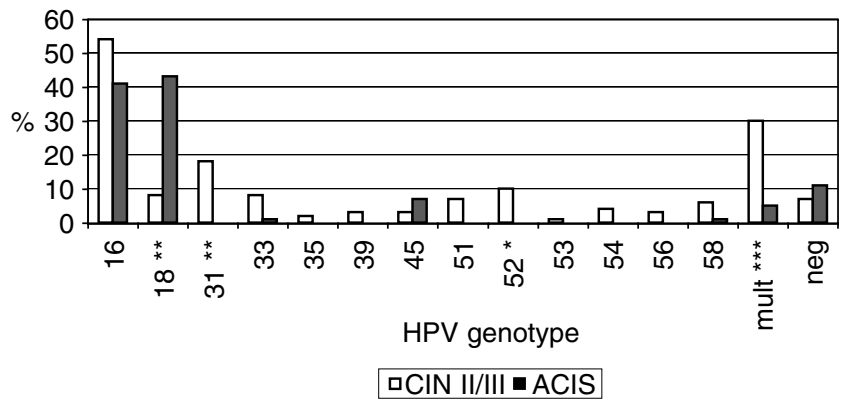

Figure I Relative frequency of specific hr-HPV genotypes in patients with CIN II/III (90 patients) and patients with ACIS (96 patients). $* P<0.05$, ** $P<0.01$, **** $P<0.001$. in patients with CIN II/III, while HPV 18 was significantly more often detected in patients with ACIS (with or without coexisting CIN). Among patients with CIN II/III, 13 different hr-HPV genotypes were detected, compared with only five different hrHPV genotypes in patients with ACIS with or without coexisting CIN.

HPV 16 was the most frequently detected HPV genotype in patients with CIN II/III (54\%), while both HPV 16 and 18 showed a high frequency of, respectively, 41 and $43 \%$ in all patients with ACIS (see Figure 1). HPV 16 or 18 were present in $79 \%$ of all patients with ACIS (five patients had a double infection with HPV 16 and 18), and in $61 \%$ of the patients with CIN II/III (one patient had a double infection with HPV 16 and 18).

Table 2 shows that there were no significant differences in the frequency of HPV 16, 45, and hr-HPV-negative patients between patients with CIN II/III and patients with ACIS and coexisting CIN. The frequency of HPV 18 was significantly lower, and the frequency of HPV 31, multiple hr-HPV, and hr-HPV genotypes other than HPV 16, 18, 31, and 45 was significantly higher in patients with CIN II/III compared with patients with ACIS and coexisting CIN. This means that the frequency of HPV 18 is significantly higher in CIN lesions coexisting with ACIS than in solitary CIN lesions.

Table 3 shows that there were no significant differences in the frequency of HPV 16, 18, 31, 45, multiple hr-HPV, and other hrHPV genotypes between patients with ACIS without coexisting CIN and patients with ACIS and coexisting CIN. The number of hrHPV-negative patients was significantly higher in patients with ACIS without coexisting CIN.

Among the patients with ACIS and coexisting CIN, there were no significant differences in the frequency of specific hr-HPV genotypes regarding the different degrees of coexisting CIN.

The mean age of the patients with ACIS without CIN was 40.1 years, while the mean age of patients with ACIS and coexisting CIN

Table 2 Frequency of hr-HPV genotypes in patients with CIN II/III compared with patients with ACIS and coexisting CIN

\begin{tabular}{lccc}
\hline hr-HPV genotype & $\begin{array}{c}\text { CIN II/III } \\
\mathbf{n}=\mathbf{9 0}\end{array}$ & $\begin{array}{c}\text { ACIS with CIN } \\
\mathbf{n}=\mathbf{4 9}\end{array}$ & Significance \\
\hline 16 & $49(54 \%)$ & $19(39 \%)$ & $\mathrm{NS}$ \\
18 & $7(8 \%)$ & $23(47 \%)$ & $\chi^{2}=26.49, P<0.001$ \\
31 & $15(17 \%)$ & $0(0 \%)$ & $\chi^{2}=8.38, P<0.01$ \\
45 & $3(3 \%)$ & $5(10 \%)$ & $\mathrm{NS}$ \\
Other & $34(38 \%)$ & $2(4 \%)$ & $\chi^{2}=19.73, P<0.001$ \\
Multiple & $26(29 \%)$ & $2(4 \%)$ & $\chi^{2}=12.48, P<0.001$ \\
Total hr-HPV positive & $84(93 \%)$ & $47(96 \%)$ & $\mathrm{NS}$ \\
hr-HPV negative & $6(7 \%)$ & $2(4 \%)$ & $\mathrm{NS}$ \\
\hline
\end{tabular}

$\mathrm{ACIS}=$ adenocarcinoma in situ; $\mathrm{hr}-\mathrm{HPV}=$ high-risk human papillomavirus; $\mathrm{CIN}=$ cervical intraepithelial neoplasia; NS = not significant.

Table 3 Frequency of hr-HPV genotypes in patients with ACIS with coexisting CIN compared with patients with ACIS without coexisting CIN

\begin{tabular}{lccc}
\hline hr-HPV genotype & $\begin{array}{c}\text { ACIS } \text { with CIN } \\
\mathbf{n}=\mathbf{4 9}\end{array}$ & $\begin{array}{c}\text { ACIS without CIN } \\
\mathbf{n = 4 7}\end{array}$ & Significance \\
\hline 16 & $19(39 \%)$ & $20(43 \%)$ & $\mathrm{NS}$ \\
18 & $23(47 \%)$ & $18(38 \%)$ & $\mathrm{NS}$ \\
31 & $0(0 \%)$ & $0(0 \%)$ & $\mathrm{NS}$ \\
45 & $5(10 \%)$ & $2(4 \%)$ & $\mathrm{NS}$ \\
Other & $2(4 \%)$ & $1(2 \%)$ & $\mathrm{NS}$ \\
Multiple & $2(4 \%)$ & $3(6 \%)$ & $\chi^{2}=6.07, P<0.02$ \\
Total hr-HPV positive & $47(96 \%)$ & $38(81 \%)$ & $\chi^{2}=6.63, P<0.01$ \\
hr-HPV negative & $2(4 \%)$ & $9(19 \%)$ & \\
\hline
\end{tabular}

$\mathrm{ACIS}=$ adenocarcinoma in situ; $\mathrm{hr}-\mathrm{HPV}=$ high-risk human papillomavirus; $\mathrm{CIN}=$ cervical intraepithelial neoplasia; NS = not significant. 
was 36.3 years $(t=2.32, P<0.05)$. There was no difference between the mean age of the total group of patients with ACIS and the patients with CIN II/III (38.2 and 37.6 years, respectively).

Within the group of patients with CIN II/III, there were no significant differences in the frequency of HPV 16, 18, 31, and 45, multiple hr-HPV, other hr-HPV genotypes, or hr-HPV-negative patients between patients with moderate dysplasia, severe dysplasia, or carcinoma in situ. Multiple hr-HPV genotypes were detected in, respectively, 22,28 , and $33 \%$ of these patients.

High-risk human papillomavirus was detected in 33 of the 37 patients $(89 \%)$ with a cervical scrape that indicated a high-grade glandular lesion, in 92 of the 99 patients (93\%) with a cervical scrape that indicated a high-grade squamous lesion, in all 22 patients (100\%) with a cervical scrape that indicated both a high-grade glandular and squamous lesion, and in 13 of the 15 patients $(87 \%)$ with a cervical scrape that indicated only a low-grade lesion. There were no significant differences in the frequency of HPV 16, 18, 31, 45, multiple or other HPV genotypes regarding the presence or absence of a suspicion of a high-grade squamous and/or glandular lesion in the cervical scrapes.

\section{DISCUSSION}

The number of patients with ACIS and coexisting CIN lesions among all patients with ACIS in this study is similar to the numbers reported in the literature (Table 1). No significant differences in the frequency of different hr-HPV genotypes were observed between patients with ACIS with coexisting CIN, and patients with ACIS without coexisting CIN, as has been reported previously (Anciaux et al, 1997; Riethdorf et al, 2000; Zaino, 2002). However, significant differences in the frequency of HPV 18, HPV 31, multiple hr-HPV genotypes, and hr-HPV genotypes other than HPV 16, 18, 31, and 45 were observed between patients with CIN II/III and patients with ACIS, either with or without coexisting CIN. One may hypothesise that coexisting glandular and squamous lesions share a common aetiology, different from solitary squamous lesions, under the influence of specific hr-HPV genotypes, as has been previously described for squamous lesions (Burghardt and Ostor, 1983; Park et al, 1998; Bekkers et al, 2002b). Indeed, in 41 of the 49 patients $(84 \%)$ in the present study the ACIS lesion was adjacent to the coexisting squamous lesion. Furthermore, Colgan and Lickrish (1990) found no differences in the linear extend, circumferential extend, multifocality, or the co-presence of invasive adenocarcinoma between patients with ACIS without CIN and ACIS with coexisting CIN lesions. These observations seem to support the hypothesis that ACIS and coexisting CIN lesions share a common aetiology.

Only five different hr-HPV genotypes (HPV 16, 18, 33, 45, 58) were detected in patients with ACIS (of which HPV 33 and 58 in $<2 \%$ of the patients), compared with 13 different hr-HPV genotypes in patients with CIN II/III. Other authors also found a limited number of hr-HPV genotypes (three to seven) among patients with ACIS (Pirog et al, 2000; Madeleine et al, 2001). This may suggest that only certain hr-HPV genotypes are able to infect and/or induce lesions in the glandular mucosa. This fact may be the reason for the significantly lower detection rate of multiple hr-HPV genotypes, and the significantly higher detection rate of HPV 18 in the cervix of patients with ACIS. However, higher rates $(13-22 \%)$ of multiple hr-HPV infections among patients with ACIS have been described in the literature (Pirog et al, 2000; Madeleine et al, 2001). The lower rate of multiple hr-HPV genotypes in the present study is probably the result of the very restrictive diagnostic criteria, and the consensus agreement by three pathologists, that was used to diagnose ACIS.
The high frequency of a single hr-HPV genotype in patients with ACIS compared with patients with CIN II/III may reflect the monoclonal aspect of the ACIS lesion, as is often the case in invasive carcinomas (Walboomers et al, 1999). Patients with (squamous) CIS in this study showed a higher prevalence (33\%) of multiple hr-HPV genotypes, as well as a higher number of different hr-HPV genotypes (12), which may be regarded as another indication that ACIS lesions may have a biologic behaviour and/ or aetiology that is different from squamous in situ lesions (Schoolland et al, 2002).

The number of hr-HPV-negative patients with ACIS without CIN in this study was significantly higher than in patients with ACIS and coexisting CIN. ACIS may be a precursor of different subtypes of invasive adenocarcinoma (like small cell or endometrioid adenocarcinoma) that have a lower or no prevalence of hrHPV (Lee, 1999; Pirog et al, 2000; Schoolland et al, 2002). These subtypes are rare, and distinction on a biopsy with only ACIS proved to be difficult (Lee, 1999; Schoolland et al, 2002). It is possible that these hr-HPV-negative subtypes of ACIS are less often associated with coexisting CIN, resulting in a higher rate of hr-HPV-negative patients among patients with ACIS without CIN.

The significant difference in age between patients with ACIS without CIN and patients with ACIS with coexisting CIN, in which the latter are younger, has been described previously (Colgan and Lickrish, 1990; Pirog et al, 2000). Different explanations for this age difference are possible. Firstly, the lesions in patients with ACIS and coexisting CIN may show a faster progression, leading to detection at a younger age. This has been described previously for HPV 18-related lesions (Barnes et al, 1988), but we did not find a difference in HPV 18 prevalence between patients with ACIS alone or ACIS and coexisting CIN. Secondly, the involvement of the squamous epithelium may be the reason for the detection of ACIS with coexisting $\mathrm{CIN}$ at a younger age, since ecto-cervical lesions are more easily detected. Thirdly, it may be a coincidental finding especially since we did not find a difference in mean age between patients with CIN II/III and the total group of patients with ACIS.

There was no significant difference in the overall sensitivity of the preceding cervical scrape for the detection of either ACIS or CIN II/III in the present study (respectively 93 and 90\%). The observed sensitivity to detect ACIS was higher than reported in the literature (Lee, 1999; Schoolland et al, 2002). However, these studies used cervical scrapes from a cervical cancer screening programme, while in the present study several patients had more than one preceding cervical scrape taken, which may have alerted the pathologist, leading to the higher sensitivity.

We did not find a relation between the presence of certain hr-HPV genotypes in the biopsy, and the sensitivity of the cervical scrape in detecting ACIS and/or CIN II/III lesions. This indicates that the detection of a lesion in the cervical scrape is not influenced by the genotype of hr-HPV infecting that lesion.

In conclusion, patients with ACIS have significantly more often HPV 18 infections, while patients with CIN II/III have significantly more often infections with HPV 31, HPV genotypes other than 16, 18, 31, and 45, and multiple hr-HPV genotypes. The detection of high-grade glandular and/or squamous lesion by cervical scrapes is not influenced by the hr-HPV genotype associated with the lesion. Among patients with ACIS, patients with coexisting CIN lesions tend to be younger, but they have a similar frequency of different hr-HPV genotypes than patients with ACIS without CIN, while the frequency of specific hr-HPV genotypes differs significantly from those of patients with CIN II/III without ACIS. These findings suggest that squamous lesions, coexisting with high-grade glandular lesions, are aetiologically different from squamous lesions without coexisting glandular lesions. The clinical implication of these findings needs further study. 


\section{REFERENCES}

Anciaux D, Lawrence WD, Gregoire L (1997) Glandular lesions of the uterine cervix: prognostic implications of human papillomavirus status. Int J Gynecol Pathol 16: $103-110$

Barnes W, Delgado G, Kurman RJ, Petrilli ES, Smith DM, Ahmed S, Lorincz AT, Temple GF, Jenson AB, Lancaster WD (1988) Possible prognostic significance of human papillomavirus type in cervical cancer. Gynecol Oncol 29: $267-273$

Bekkers RL, Melchers WJ, Bakkers JM, Hanselaar AG, Quint WG, Boonstra $\mathrm{H}$, Massuger LF (2002a) The role of genotype-specific human papillomavirus detection in diagnosing residual cervical intraepithelial neoplasia. Int J Cancer 102: $148-151$

Bekkers RL, Melchers WJ, Bulten J, Boonstra H, Quint WG, Hanselaar AG, Massuger LF (2002b) Localized distribution of human papillomavirus genotypes in the uterine cervix. Eur J Gynaecol Oncol 23: 203-206

Bosch FX, Lorincz A, Munoz N, Meijer CJ, Shah KV (2002) The causal relation between human papillomavirus and cervical cancer. J Clin Pathol 55: 244-265

Burghardt E, Ostor AG (1983) Site and origin of squamous cervical cancer: a histomorphologic study. Obstet Gynecol. 62: 117-127

Colgan TJ, Lickrish GM. (1990) The topography and invasive potential of cervical adenocarcinoma in situ, with and without associated squamous dysplasia. Gynecol Oncol 36: 246-249

Duggan MA, McGregor SE, Benoit JL, Inoue M, Nation JG, Stuart GC (1995) The human papillomavirus status of invasive cervical adenocarcinoma: a clinicopathological and outcome analysis. Hum Pathol 26: 319-325

Higgins GD, Phillips GE, Smith LA, Uzelin DM, Burrell CJ (1992) High prevalence of human papillomavirus transcripts in all grades of cervical intraepithelial glandular neoplasia. Cancer 70: 136-146

Kleter B, van Doorn LJ, ter Schegget J, Schrauwen L, van Krimpen K, Burger M, ter Harmsel B, Quint W (1998) Novel short-fragment PCR assay for highly sensitive broad-spectrum detection of anogenital human papillomaviruses. Am J Pathol 153: 1731 - 1739

Kleter B, van Doorn LJ, Schrauwen L, Molijn A, Sastrowijoto S, ter Schegget J, Lindeman J, ter Harmsel B, Burger M, Quint W (1999) Development and clinical evaluation of a highly sensitive PCR-reverse hybridization line probe assay for detection and identification of anogenital human papillomavirus. J Clin Microbiol 37: 2508-2517

Leary J, Jaworski R, Houghton R (1991) In-situ hybridization using biotinylated DNA probes to human papillomavirus in adenocarcinomain-situ and endocervical glandular dysplasia of the uterine cervix. Pathology 23: $85-89$

Lee KR (1999) Adenocarcinoma in situ with a small cell (endometrioid) pattern in cervical smears: a test of the distinction from benign mimics using specific criteria. Cancer 87: 254-258

Madeleine MM, Daling JR, Schwartz SM, Shera K, McKnight B, Carter JJ, Wipf GC, Critchlow CW, McDougall JK, Porter P, Galloway DA (2001)
Human papillomavirus and long-term oral contraceptive use increase the risk of adenocarcinoma in situ of the cervix. Cancer Epidemiol Biomarkers Prev 10: $171-177$

McLachlin CM, Shen LH, Sheets EE, Kozakewich H, Perlman SE, Tate JE, Crum CP (1997) Disparities in mean age and histologic grade between human papillomavirus type specific early cervical neoplasms. Hum Pathol 28: 1226 - 1229

Melchers WJ, Bakkers JM, Wang J, de Wilde PC, Boonstra H, Quint WG, Hanselaar AG (1999) Short fragment polymerase chain reaction reverse hybridization line probe assay to detect and genotype a broad spectrum of human papillomavirus types. Clinical evaluation and follow-up. Am J Pathol 155: $1473-1478$

Park J, Sun D, Genest DR, Trivijitsilp P, Suh I, Crum CP (1998) Coexistence of low and high grade squamous intraepithelial lesions of the cervix: morphologic progression or multiple papillomaviruses? Gynecol Oncol 70: $386-391$

Pirog EC, Kleter B, Olgac S, Bobkiewicz P, Lindeman J, Quint WG, Richart RM, Isacson C (2000) Prevalence of human papillomavirus DNA in different histological subtypes of cervical adenocarcinoma. Am J Pathol 157: $1055-1062$

Quint WG, Scholte G, van Doorn LJ, Kleter B, Smits PH, Lindeman J (2001) Comparative analysis of human papillomavirus infections in cervical scrapes and biopsy specimens by general SPF(10) PCR and HPV genotyping. J Pathol 194: $51-58$

Riethdorf L, Riethdorf S, Lee KR, Cviko A, Loening T, Crum CP (2002) Human papillomaviruses, expression of $\mathrm{p} 16^{\mathrm{INK}}$, and endocervical glandular neoplasia. Hum Pathol 33: 899-904

Riethdorf S, Riethdorf L, Milde-Langosch K, Park TW, Loning T (2000) Differences in HPV 16- and HPV 18 E6/E7 oncogene expression between in situ and invasive adenocarcinomas of the cervix uteri. Virchows Arch 437: $491-500$

Schoolland M, Segal A, Allpress S, Miranda A, Frost FA, Sterrett GF (2002) Adenocarcinoma in situ of the cervix. Cancer 96: $330-337$

Walboomers JM, Jacobs MV, Manos MM, Bosch FX, Kummer JA, Shah KV, Snijders PJ, Peto J, Meijer CJ, Munoz N (1999) Human papillomavirus is a necessary cause of invasive cervical cancer worldwide. J Pathol 189: $12-19$

Wells M, Brown LJ (1986) Glandular lesions of the uterine cervix: the present state of our knowledge. Histopathology 10: 777-792

Wright Jr TC, Cox JT, Massad LS, Twiggs LB, Wilkinson EJ (2002) 2001 Consensus Guidelines for the management of women with cervical cytological abnormalities. JAMA 287: 2120-2129

Zaino RJ (2002) Symposium part I: adenocarcinoma in situ, glandular dysplasia, and early invasive adenocarcinoma of the uterine cervix. Int J Gynecol Pathol 21: 314-326 